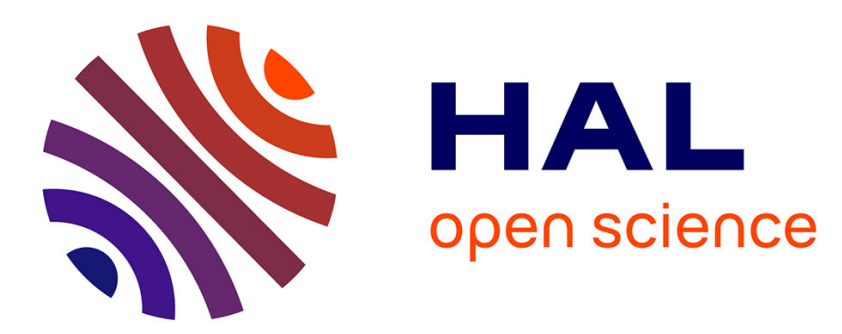

\title{
Relaxation effects in Mössbauer transmission and scattering. I general formulas
}

\author{
F. Hartmann-Boutron
}

\section{To cite this version:}

F. Hartmann-Boutron. Relaxation effects in Mössbauer transmission and scattering. I general formulas. Journal de Physique, 1976, 37 (5), pp.537-548. 10.1051/jphys:01976003705053700 . jpa00208448

\section{HAL Id: jpa-00208448 https://hal.science/jpa-00208448}

Submitted on 1 Jan 1976

HAL is a multi-disciplinary open access archive for the deposit and dissemination of scientific research documents, whether they are published or not. The documents may come from teaching and research institutions in France or abroad, or from public or private research centers.
L'archive ouverte pluridisciplinaire HAL, est destinée au dépôt et à la diffusion de documents scientifiques de niveau recherche, publiés ou non, émanant des établissements d'enseignement et de recherche français ou étrangers, des laboratoires publics ou privés. 


\author{
Classification \\ Physics Abstracts \\ $4.200-8.610-8.680$
}

\title{
RELAXATION EFFECTS IN MÖSSBAUER TRANSMISSION AND SCATTERING I GENERAL FORMULAS
}

\author{
F. HARTMANN-BOUTRON \\ Laboratoire de Physique des Solides $\left({ }^{*}\right)$ \\ Université Paris-Sud, 91405 Orsay, France \\ and \\ Laboratoire de Spectrométrie Physique $\left(^{*}\right)$ \\ U.S.M.G., B.P. 53, 38041 Grenoble Cedex (France) $\left({ }^{* *}\right)$
}

(Reçu le 6 octobre 1975, accepté le 9 janvier 1976)

\begin{abstract}
Résumé. - Dans cette première partie (I) nous établissons quelques formules générales pour la transmission et la diffusion Mössbauer en l'absence de relaxation. Nous discutons en détail l'influence de la largeur de raie du rayonnement incident sur les observables physiques. Nous examinons aussi les règles de sélection angulaires pour la diffusion par une poudre. Dans la seconde partie (II) nous introduirons la relaxation et comparerons les avantages et les inconvénients des différentes techniques Mössbauer dans les études de relaxation.

Abstract. - In this first part (I) we derive some general formulae for Mössbauer transmission and scattering in the absence of relaxation. Emphasis is put on the influence of the linewidth of the incident radiation on physical observables. We also examine the angular selection rules for scattering by a powder. In the second part (II) we will introduce relaxation and compare the advantages and limitations of the different Mössbauer techniques in relaxation studies.
\end{abstract}

1. Introduction. - In a previous series of papers [1-4] we considered the effect of relaxation on the Mössbauer spectrum of an emitter. It was assumed that the Mössbauer transition was the second step of a radioactive cascade $I_{i} \stackrel{k_{i}}{\rightarrow} I \stackrel{k}{\rightarrow} I_{g}$. In that case the Mössbauer lineshape of a powder (or of a single crystal with isotropic h.f.s.) is given by $[2,4]$ :

$$
I(\omega) \propto \int_{0}^{\infty} \mathrm{d} \tau \underset{\text { atom }}{\operatorname{Trace}}\left\{\mathbf{T}_{L}(0) \cdot \mathbf{T}_{L}^{+}(\tau) \sigma^{\mathrm{I}}(1 / \Gamma)\right\} \mathrm{e}^{(i \omega-\Gamma / 2) \tau}
$$

where $\sigma^{\mathrm{I}}(1 / \Gamma)$ is the average density matrix of the nuclear state $\mathbf{I}$ at the time of $\gamma$ emission and $\mathbf{T}_{L}$ the nuclear moment responsible for the $\gamma$ transition.

This formula is strictly valid only in the absence of relaxation. In reference [4] we have shown how relaxation effects can be introduced into the problem. Assuming that the excited electronuclear state $I$ is described by an orthonormal set of kets $|f\rangle$ and that

$\left({ }^{*}\right)$ Associés au C.N.R.S.

$(* *)$ New address. the electronuclear state $\mathrm{I}_{g}$ is described by kets $|g\rangle$ the final expression for the lineshape is :

$$
\begin{aligned}
I(\omega) \propto \operatorname{Re} & \sum_{\substack{f_{1} f_{2} f_{3} \\
f_{4}, f_{5}, g_{1}, g_{2}}}\left\langle f_{1}\left|\mathbf{T}_{L}\right| g_{1}\right\rangle\left\langle g_{2}\left|\mathbf{T}_{L}^{+}\right| f_{2}\right\rangle \times \\
& \times\left\langle g_{1} f_{3}\left|\frac{-1}{i \omega-\frac{\Gamma}{2}+\frac{i}{\hbar} \mathcal{H}_{0}^{\times}+R}\right| g_{2} f_{2}\right\rangle \\
& \times\left(\left\langle f_{3} f_{1}\left|\frac{-1}{-\Gamma-\frac{i}{\hbar} \mathcal{H}_{0}^{\times}+S}\right| f_{4} f_{5}\right\rangle\right. \\
& \left.\times\left\langle f_{4}\left|\sigma_{\text {in }}^{\mathrm{I}}\right| f_{5}\right\rangle\right)
\end{aligned}
$$

where $\sigma_{\text {in }}^{\mathrm{I}}$ is the initial value of $\sigma^{\mathrm{I}}$ just after being fed by the radioactive decay of $I_{i}$ and relaxation effects are contained into the relaxation matrices $S$ and $R=S_{T}[4]$. It appears that the Mössbauer lineshape of an emitter contains two Liouville matrices. In the case of $\mathrm{Yb}^{170} \mathrm{Au}$ where the hyperfine hamiltonian $\mathcal{H}_{0}$ is much larger than the inverse nuclear lifetime $\Gamma$, the first Liouville matrix controls the lineshape 
anomalies induced by relaxation at temperatures $k_{\mathrm{B}} T>\mathscr{H}_{0}$, while the second Liouville matrix controls the line intensity anomalies observed at temperatures $k_{\mathrm{B}} T<\mathcal{H}_{0}$; these two type of anomalies correspond respectively to the condition $S \gtrsim \mathscr{H}_{0}$ (lineshape) and $S \lesssim \Gamma$ (intensities).

In the present paper we shall extend our theory to the problem of Mössbauer transmission and scattering. Scattering was already studied in two recent papers by Balko and Hoy [5, 6] and in another one by Afanasev and Gorobchenko [7]. Here we shall discard all difficulties connected with interference or thickness effects and only investigate the potentialities of Mössbauer scattering as a tool for relaxation studies.

Let us compare the emitter and the scatterer. In the case of an emitter the two successive radioactive transitions are due to spontaneous emission which has an isotropic character and can be considered as being induced by vacuum fluctuations with a white spectrum. In an absorber there are also two nuclear transitions, first, induced absorption of an incident photon $\mathbf{k}_{\mathbf{i}}\left(\mathrm{I}_{g} \stackrel{\mathbf{k}_{\mathbf{i}}}{\rightarrow} \mathrm{I}\right)$ and, second, spontaneous emission of a scattered photon $\mathbf{k}\left(\mathrm{I} \stackrel{\mathbf{k}}{\rightarrow} \mathrm{I}_{g}\right)$. However the excitation process is not isotropic; moreover, instead of a white spectrum the incident radiation has a lorentzian frequency distribution, with a width $\Gamma^{\prime}$ which is comparable to the line width of the absorber, so that the absorption and emission processes are not really well separated. Finally we must distinguish between :

a) Coherent elastic scattering :

$\alpha$ ) forward scattering, which gives rise to a refraction index which depends on the average value $\left\langle V_{\text {diff }}\right\rangle$ of the scattering amplitude $V_{\text {diff }}$ (the average being taken over the atomic variables);

$\beta$ ) lateral Rayleigh scattering proportional to $\left\langle V_{\text {diff }}\right\rangle^{2}$.

b) Incoherent scattering proportional to :

$$
\left\langle\left(V_{\text {diff }}-\left\langle V_{\text {diff }}\right\rangle\right)^{2}\right\rangle \text {. }
$$

What is detected in a Mössbauer scattering expe riment is the sum of the elastic and inelastic scattering, which is proportional to $\left\langle\left(V_{\text {diff }}\right)^{2}\right\rangle$.

In what follows we shall first analyse the radiative processes and derive the formulae for Mössbauer transmission and scattering in the absence of relaxation. Attention will be paid to the selection rules. Then in part II we will introduce the relaxation and compare the informations which can be derived from the three types of Mössbauer experiments.

2. General equations for the scattering process. This analysis will proceed along the same lines as the cascade calculation of reference [8].
2.1 Notations. - The interaction between the electromagnetic field and the nucleus is :

$\mathcal{H}_{\mathbf{R}}=\sum_{\mathbf{k}, \lambda}\left(\frac{2 \pi \hbar c}{L^{3} k}\right)^{1 / 2}\left\{H_{\mathbf{a}}\left(\mathbf{k}, \varepsilon_{\lambda}\right) a_{\mathbf{k} \lambda}+H_{\mathbf{a}}^{+}\left(\mathbf{k}, \varepsilon_{\lambda}\right) a_{\mathbf{k} \lambda}^{+}\right\}$

with :

$$
H_{\mathrm{a}}\left(\mathbf{k}, \varepsilon_{\lambda}\right)=-\sum_{L M q} q^{\langle\pi\rangle} T_{L M}^{\langle\pi\rangle} \mathfrak{D}_{M q}^{L}(\mathrm{R})
$$

where $a_{\mathbf{k} \lambda}^{+}$is the creation operator of a photon with wave vector $\mathbf{k}$ and polarization $\lambda$ (quantized in a box $L^{3}$ ), $T_{L M}$ is one of the components of the multipole moment $\mathbf{T}_{L}$ of the nucleus, $q= \pm 1$ and $\mathfrak{D}_{M q}^{L}(\mathrm{R})$ is a rotation matrix. In reference [8] we adopted the conventions of Edmonds. Then :

$$
\mathfrak{D}_{M q}^{L}(\mathrm{R})=\mathfrak{D}_{M q}^{L}(-\varphi,-\theta, 0)
$$

where $\theta, \varphi$ are the polar angles of the photon with respect to a frame attached to the atom. On the contrary, in Messiah's notations :

$$
\mathfrak{D}_{M q}^{L}(\mathrm{R})=\mathfrak{D}_{M q}^{L}(\varphi, \theta, 0) .
$$

In the absence of relaxation the atom is described by a hamiltonian $\mathscr{H}_{\mathrm{N}}+\mathscr{H}_{0}$ where the nuclear hamiltonian $\mathscr{H}_{\mathrm{N}}$ defines the nuclear levels $\mathrm{I}, \mathrm{I}_{g}$ with

$$
E_{\mathrm{I}}-E_{\mathbf{I}_{g}}=\hbar \Omega
$$

while $\mathscr{H}_{0}$ splits these levels into electronuclear states $|f\rangle,|g\rangle^{\prime}\left({ }^{1}\right)$ :

$$
\begin{aligned}
& \left(\mathscr{H}_{\mathbf{N}}+\mathscr{H}_{0}\right)|f\rangle=\hbar\left(\Omega+\omega_{f}\right)|f\rangle \\
& \left(\mathscr{H}_{\mathbf{N}}+\mathscr{H}_{0}\right)|g\rangle=\hbar \omega_{g}|g\rangle .
\end{aligned}
$$

As for the free radiation field, its hamiltonian is :

$$
\mathscr{H}_{\mathbf{L}}=\sum_{\mathbf{k} \lambda} \hbar \omega_{\mathbf{k} \lambda} a_{\mathbf{k} \lambda}^{+} a_{\mathbf{k} \lambda}
$$

The physical states of interest in the scattering process are :

$$
\begin{aligned}
& \left.\mid g, \text { incident photon } \mathbf{k}_{\mathbf{i}} \lambda_{\mathbf{i}}\right\rangle \\
& \mid f, \text { zero photon }\rangle \\
& \mid g, \text { scattered photon } \mathbf{k} \lambda\rangle
\end{aligned}
$$

or alternatively to parallel the notations of reference [9] :

$$
\begin{aligned}
& |g\rangle \\
& \left|f,-\mathbf{k}_{\mathbf{i}} \lambda_{\mathbf{i}}\right\rangle \\
& \left|g,-\mathbf{k}_{\mathbf{i}} \lambda_{\mathbf{i}}, \mathbf{k} \lambda\right\rangle .
\end{aligned}
$$

( $\left.{ }^{1}\right)$ In eq. (1), $\omega=\omega_{k}-\Omega$ where $\omega_{k}$ is the frequency of the photon. 
We define, in the interaction representation :

and

$$
\widetilde{\mathscr{H}}_{\mathbf{R}}(t)=U^{+}(t) \mathscr{H}_{\mathbf{R}} U(t)=\exp \left[\frac{i}{\hbar}\left(\mathscr{H}_{0}+\mathscr{H}_{\mathbf{N}}+\mathscr{H}_{\mathbf{L}}\right) t\right] \mathscr{H}_{\mathbf{R}} \times \exp \left[-\frac{i}{\hbar}\left(\mathscr{H}_{0}+\mathscr{H}_{\mathbf{N}}+\mathscr{H}_{\mathbf{L}}\right) t\right]
$$

$$
\widetilde{\psi}(t)=\sum_{g} b_{g}|g\rangle+\sum_{f} b_{f,-\mathbf{k}_{\mathbf{i}} \lambda_{\mathbf{i}}}\left|f,-\mathbf{k}_{\mathbf{i}} \lambda_{\mathbf{i}}\right\rangle+\sum_{g, \mathbf{k} \lambda} b_{g,-\mathbf{k}_{\mathbf{i}} \lambda_{\mathbf{i}}, \mathbf{k} \lambda}\left|g,-\mathbf{k}_{\mathbf{i}} \lambda_{\mathbf{i}}, \mathbf{k} \lambda\right\rangle
$$

$\widetilde{\psi}(t)$ is the solution of :

$$
i \hbar \frac{\partial \widetilde{\psi}}{\partial t}=\breve{\mathscr{H}}_{\mathbf{R}}(t) \widetilde{\psi}(t)
$$

2.2. Coupled equations AND THeir integration. - Eq. (12) is equivalent to the system :

$$
\begin{aligned}
& i \dot{b}_{g}=\frac{1}{\hbar} \sum_{f}\left\langle g\left|\check{\mathscr{H}}_{\mathbf{R}}(t)\right| f,-\mathbf{k}_{\mathbf{i}} \lambda_{\mathbf{i}}\right\rangle b_{f,-\mathbf{k}_{\mathbf{i}} \lambda_{\mathbf{i}}} \\
& i \dot{b}_{f,-\mathbf{k}_{\mathbf{i}} \lambda_{\mathrm{i}}}=\frac{1}{\hbar} \sum_{\substack{g \\
\mathbf{k} \lambda}}\left\langle f,-\mathbf{k}_{\mathbf{i}} \lambda_{\mathrm{i}}\left|\breve{\mathscr{H}}_{\mathbf{R}}(t)\right| g\right\rangle b_{g}+\frac{1}{\hbar} \sum_{\substack{g \\
\mathbf{k} \lambda}}\left\langle f,-\mathbf{k}_{\mathbf{i}} \lambda_{\mathbf{i}}\left|\breve{\mathscr{H}}_{\mathbf{R}}(t)\right| g,-\mathbf{k}_{\mathbf{i}} \lambda_{\mathbf{i}}, \mathbf{k} \lambda\right\rangle b_{g,-\mathbf{k}_{\mathbf{i}} \lambda_{\mathbf{i}}, \mathbf{k} \lambda} \\
& i \dot{b}_{g,-\mathbf{k}_{\mathbf{i}} \lambda_{i}, \mathbf{k} \lambda}=\frac{1}{\hbar} \sum_{f}\left\langle g,-\mathbf{k}_{\mathbf{i}} \lambda_{\mathbf{i}}, \mathbf{k} \lambda\left|\breve{\mathscr{H}}_{\mathbf{R}}(t)\right| f,-\mathbf{k}_{\mathbf{i}} \lambda_{\mathbf{i}}\right\rangle b_{f,-\mathbf{k}_{\mathbf{i}} \lambda_{\mathrm{i}}} .
\end{aligned}
$$

The summation over $\mathbf{k} \lambda$ in the second of these equations is associated with the occurrence of spontaneous emission, whose effect is to give to all levels a damping $\Gamma$ and a self energy $\Delta E$. These quantities can be computed in exactly the same way as in reference [8]. Dropping the self energy term, eq. (14) can then be replaced by :

$$
\dot{i b}_{f,-\mathbf{k}_{\mathbf{i}} \lambda_{\mathrm{i}}}=-\frac{\Gamma}{2} b_{f,-\mathbf{k}_{\mathbf{i}} \lambda_{\mathrm{i}}}+\frac{1}{\hbar} \sum_{g}\left\langle f,-\mathbf{k}_{\mathbf{i}} \lambda_{\mathbf{i}}\left|\breve{\mathscr{H}}_{\mathbf{R}}(t)\right| g\right\rangle b_{g}(t) .
$$

Let us now relate $\dot{b}_{g,-\mathbf{k}_{\mathrm{i}} \lambda_{\mathrm{i}}, \mathbf{k} \lambda}$ to $b_{g}$. For this we first integrate eq. (16) :

$$
i b_{f,-\mathbf{k}_{\mathrm{i}} \lambda_{\mathrm{i}}}=\frac{1}{\hbar} \int_{t_{0}}^{t} \exp \left[-\frac{\Gamma}{2}\left(t-t^{\prime}\right)\right] \sum_{g}\left\langle f,-\mathbf{k}_{\mathrm{i}} \lambda_{\mathrm{i}}\left|\breve{\mathscr{H}}_{\mathbf{R}}\left(t^{\prime}\right)\right| g\right\rangle b_{g}\left(t^{\prime}\right)
$$

and we insert it into eq. (15):

$$
\begin{aligned}
\dot{b}_{g^{\prime},-\mathbf{k}_{\mathrm{i}} \lambda_{\mathrm{i}}, \mathbf{k} \lambda}=-\frac{1}{\hbar^{2}} \sum_{f, g} \int_{t_{0}}^{t} \exp \left[-\frac{\Gamma}{2}\left(t-t^{\prime}\right)\right]\left\langle g^{\prime},-\mathbf{k}_{\mathrm{i}} \lambda_{\mathrm{i}}, \mathbf{k} \lambda\left|\breve{\mathscr{H}}_{\mathrm{R}}(t)\right| f,-\mathbf{k}_{\mathrm{i}} \lambda_{i}\right\rangle \times & \times \\
\times & \left.<f,-\mathbf{k}_{\mathrm{i}} \lambda_{\mathrm{i}}\left|\widetilde{\mathscr{H}}_{\mathbf{R}}\left(t^{\prime}\right)\right| g\right\rangle b_{g}\left(t^{\prime}\right) .
\end{aligned}
$$

This expression will be our starting point for the computation of the Mössbauer transmission in the next paragraph.

In the case of lateral scattering we will need the number of scattered photons $\mathbf{k} \lambda$. This is given by :

$$
\begin{aligned}
& \frac{\mathrm{d}}{\mathrm{d} t} \sum_{g}\left|b_{g,-\mathbf{k}_{\mathbf{i}} \lambda_{i}, \mathbf{k} \lambda}\right|^{2} \equiv \sum_{\boldsymbol{g}}\left(\dot{b}_{g,-\mathbf{k}_{\mathrm{i}} \lambda_{\mathrm{i}}, \mathbf{k} \lambda} b_{g,-\mathbf{k}_{\mathrm{i}} \lambda_{i}, \mathbf{k} \lambda}^{*}+\text { c.c. }\right)= \\
& =\frac{1}{\hbar^{4}} \sum_{\substack{g, g^{\prime}, g^{\prime \prime} \\
f^{\prime \prime} f^{\prime \prime}}} \int_{t_{0}}^{t} \mathrm{~d} t^{\prime} \int_{t_{0}}^{t} \mathrm{~d} t^{\prime \prime} \int_{t_{0}}^{t^{\prime \prime}} \mathrm{d} t^{\prime \prime \prime} \exp \left[-\frac{\Gamma}{2}\left(t-t^{\prime}\right)\right] \cdot \exp \left[-\frac{\Gamma}{2}\left(t^{\prime \prime}-t^{\prime \prime \prime}\right)\right] \times \\
& \times\left\langle g,-\mathbf{k}_{\mathrm{i}} \lambda_{\mathrm{i}}, \mathbf{k} \lambda\left|\breve{\mathscr{K}}_{\mathbf{R}}(t)\right| f^{\prime},-\mathbf{k}_{\mathrm{i}} \lambda_{\mathrm{i}}\right\rangle\left\langle f^{\prime},-\mathbf{k}_{\mathrm{i}} \lambda_{\mathrm{i}}\left|\breve{\mathscr{H}}_{\mathbf{R}}\left(t^{\prime}\right)\right| g^{\prime}\right\rangle b_{g^{\prime}}\left(t^{\prime}\right) b_{g^{\prime \prime}}^{*}\left(t^{\prime \prime \prime}\right) \\
& \times\left\langle g^{\prime \prime}\left|\breve{\mathscr{H}}_{\mathbf{R}}\left(t^{\prime \prime \prime}\right)\right| f^{\prime \prime},-\mathbf{k}_{\mathbf{i}} \lambda_{\mathbf{i}}\right\rangle\left\langle f^{\prime \prime},-\mathbf{k}_{\mathbf{i}} \lambda_{\mathbf{i}}\left|\breve{\mathscr{H}}_{\mathbf{R}}\left(t^{\prime \prime}\right)\right| g,-\mathbf{k}_{\mathbf{i}} \lambda_{\mathbf{i}}, \mathbf{k} \lambda\right\rangle+\text { c.c. }
\end{aligned}
$$

3. Application to Mössbauer transmission. - The integral in eq. (18) contains $b_{g}\left(t^{\prime}\right)$. The time variation of $b_{g}$ is due to the absorption of the incident radiation, which gives rise to a damping $1 / T_{p}$ and an energy shift $\Delta E^{\prime}$ of the ground state $|g\rangle[9] .1 / T_{p}$ and $\Delta E^{\prime}$ are proportional to the intensity of the incident radiation and depend on its wavelength (since we have a narrow line excitation). In practice the incident intensity is very low 
and $1 / T_{p}, \Delta E^{\prime} \ll \Gamma$. In eq. (18) we can therefore consider $b_{g}\left(t^{\prime}\right)$ as constant on a time scale $t-t^{\prime} \sim 1 / \Gamma$ and replace it by $b_{g}(t)$. Also we replace $t_{0}$ by $-\infty$. Eq. (18) then becomes :

with

$$
\dot{i b}_{g^{\prime},-\mathbf{k}_{\mathbf{i}} \lambda_{\mathrm{i}}, \mathbf{k} \lambda}=\frac{1}{\hbar} \sum_{g}\left\langle g^{\prime},-\mathbf{k}_{\mathrm{i}} \lambda_{\mathrm{i}}, \mathbf{k} \lambda\left|\tilde{\mathscr{H}}_{\mathrm{diff}}\right| g\right\rangle b_{g}(t)
$$

$$
\tilde{\mathscr{H}}_{\mathrm{diff}}=-\frac{i}{\hbar} \int_{-\infty}^{t} \exp \left[-\frac{\Gamma}{2}\left(t-t^{\prime}\right)\right] \mathrm{d} t^{\prime} P_{\mathrm{fi}} \tilde{\mathscr{H}}_{\mathrm{R}}(t) P_{\mathrm{ex}} \tilde{\mathscr{H}}_{\mathrm{R}}\left(t^{\prime}\right) P_{\text {in }}
$$

where we have introduced the projectors :

$$
\begin{aligned}
& P_{\mathrm{fi}} \text { on the states }\left|g,-\mathbf{k}_{\mathrm{i}} \lambda_{\mathrm{i}}, \mathbf{k} \lambda\right\rangle \\
& P_{\text {ex }} \text { on the states }\left|f,-\mathbf{k}_{\mathrm{i}} \lambda_{\mathrm{i}}\right\rangle \\
& P_{\mathrm{in}} \text { on the states }|g\rangle .
\end{aligned}
$$

Since $U(t)$, eq. (10), commutes with these projectors, we have in the normal representation :

$$
\mathscr{H}_{\mathrm{diff}}=-\frac{i}{\hbar} \int_{-\infty}^{t} \exp \left[-\frac{\Gamma}{2}\left(t-t^{\prime}\right)\right] P_{\mathrm{fi}} \mathcal{H}_{\mathrm{R}} P_{\mathrm{ex}} U\left(t-t^{\prime}\right) P_{\mathrm{ex}} \mathcal{H}_{\mathrm{R}} P_{\mathrm{in}} U\left(t-t^{\prime}\right) P_{\mathrm{in}} .
$$

Let us now write $\mathscr{T}_{\mathbf{R}}$ in explicit form, assuming monochromatic irradiation by photons $\mathbf{k}_{\mathbf{i}} \lambda_{\mathbf{i}}$, and introduce the atomic evolution operator $U_{0}(t)=\exp \left(-\frac{i}{\hbar} \mathcal{H}_{0} t\right) . \mathscr{H}_{\text {diff }}$ takes the form :

with :

$$
\mathscr{H}_{\text {diff }}=\frac{2 \pi \hbar c}{L^{3} k} a_{\mathbf{k} \lambda}^{+} a_{\mathbf{k}_{\mathbf{i}} \lambda_{\mathrm{i}}} V_{\text {diff }}\left(\mathbf{k} \lambda, \mathbf{k}_{\mathbf{i}} \lambda_{\mathrm{i}}\right)
$$

$$
\begin{aligned}
V_{\mathrm{diff}}\left(\mathbf{k} \lambda, \mathbf{k}_{\mathrm{i}} \lambda_{\mathrm{i}}\right)=-\frac{i}{\hbar} \int_{-\infty}^{t} \exp \left\{\left[-\mathrm{i}\left(\Omega-\omega_{\mathrm{i}}\right)-\frac{\Gamma}{2}\right]\left(t-t^{\prime}\right)\right\} \mathrm{d} t^{\prime} \times \\
\\
\times H_{\mathrm{a}}^{+}(\mathbf{k} \lambda) U_{0}\left(t-t^{\prime}\right) H_{\mathrm{a}}\left(\mathbf{k}_{\mathrm{i}} \lambda_{\mathrm{i}}\right) U_{0}^{+}\left(t-t^{\prime}\right) .
\end{aligned}
$$

(where $\omega_{\mathrm{i}}$ is the frequency of the incident photon).

As recalled by Blume and Kistner [10], the refraction index which characterizes the transmission of the incident $\gamma$ ray through the absorber is (for small absorption) :

$$
n=1+\frac{2 \pi}{k^{2}} N \bar{f}
$$

where the coherent forward scattering amplitude is :

$$
\bar{f}=\frac{L^{3} k}{2 \pi \hbar c}\left\langle\mathscr{H}_{\text {diff }}\right\rangle=\left\langle V_{\text {diff }}\right\rangle
$$

the average being taken over the atomic variables :

$$
\begin{aligned}
\left\langle V_{\text {diff }}\right\rangle=-\frac{i}{\hbar} \int_{-\infty}^{t} \exp \left\{\left[-i\left(\Omega-\omega_{i}\right)-\frac{\Gamma}{2}\right](t\right. & \left.\left.-t^{\prime}\right)\right\} \mathrm{d} t^{\prime} \times \\
& \times \underset{\text { at }}{\operatorname{Trace}}\left\{\sigma H_{\mathrm{a}}^{+}(\mathbf{k} \lambda) U_{0}\left(t-t^{\prime}\right) H_{\mathrm{a}}\left(\mathbf{k}_{\mathrm{i}} \lambda_{\mathrm{i}}\right) U_{0}^{+}\left(t-t^{\prime}\right)\right\}
\end{aligned}
$$

$\sigma$ is the density matrix of the ground Mössbauer state, assumed to be at thermal equilibrium : $\sigma=\sigma_{\mathrm{B}}$. Since it is constant :

$$
U_{0}^{+}\left(t-t^{\prime}\right) \sigma U_{0}\left(t-t^{\prime}\right)=\sigma .
$$

Therefore after rearranging the trace, we may write :

$$
\begin{aligned}
\left\langle V_{\text {diff }}\right\rangle=-\frac{i}{\hbar} \int_{-\infty}^{t} \exp \left\{\left[-i\left(\Omega-\omega_{\mathrm{i}}\right)-\frac{\Gamma}{2}\right]\left(t-t^{\prime}\right)\right\} \mathrm{d} t^{\prime} \times \\
\times \underset{\text { at }}{ }\left\{H_{\mathrm{a}}^{+}(\mathbf{k} \lambda) U_{0}\left(t-t^{\prime}\right) H_{\mathrm{a}}\left(\mathbf{k}_{\mathrm{i}} \lambda_{\mathrm{i}}\right) \sigma U_{0}^{+}\left(t-t^{\prime}\right)\right\} .
\end{aligned}
$$


If, instead of being monochromatic, the incident light has a lorentzian shape with width $\Gamma^{\prime}$ and central frequency $\omega_{\mathrm{i}_{0}}$, we must in this expression replace the exponential factor by :

$$
\int_{-\infty}^{+\infty} \mathrm{d} \omega_{\mathrm{i}}\left[\frac{1}{\pi} \frac{\frac{\Gamma^{\prime}}{2}}{\left(\frac{\Gamma^{\prime}}{2}\right)^{2}+\left(\omega_{\mathrm{i}}-\omega_{\mathrm{i}_{0}}\right)^{2}}\right] \exp \left\{\left[-i\left(\Omega-\omega_{\mathrm{i}}\right)-\frac{\Gamma}{2}\right]\left(t-t^{\prime}\right)\right\}
$$

which is easily shown by contour integration to be equal to :

$$
\exp \left\{\left[-i\left(\Omega-\omega_{\mathrm{i}_{0}}\right)-\frac{\Gamma+\Gamma^{\prime}}{2}\right]\left(t-t^{\prime}\right)\right\}
$$

Let us assume in addition that we irradiate with natural (unpolarized) light, that we do not detect the polarization of the transmitted light and that the nuclear transition has pure multipole character. Then we may replace $H_{\mathrm{a}}\left(\mathbf{k}_{\mathrm{i}} \lambda_{\mathrm{i}}\right)$ by $\sum_{M q} T_{L M} \mathcal{D}_{M q}^{L}\left(\theta_{\mathrm{i}} \varphi_{\mathrm{i}}\right)$ and so on. Since in forward scattering $: \theta, \varphi \equiv \theta_{\mathrm{i}} \varphi_{\mathrm{i}}$ we get :

$$
\begin{array}{r}
\left\langle V_{\text {diff }}\right\rangle=-\frac{i}{\hbar} \int_{-\infty}^{t} \exp \left\{\left[-i\left(\Omega-\omega_{\mathrm{i}_{0}}\right)-\frac{\Gamma+\Gamma^{\prime}}{2}\right]\left(t-t^{\prime}\right)\right\} \mathrm{d} t^{\prime} \sum_{\substack{M q \\
q= \pm 1}} \sum_{\substack{M^{\prime} p \\
p= \pm 1}} \mathcal{D}_{M q}^{L^{*}}\left(\theta_{\mathrm{i}} \varphi_{\mathrm{i}}\right) \times \\
\times \mathcal{D}_{M^{\prime} p}^{L}\left(\theta_{\mathrm{i}} \varphi_{\mathrm{i}}\right) \operatorname{Trace}\left[T_{L M}^{+} U_{0}\left(t-t^{\prime}\right) T_{L M^{\prime}} \sigma U_{0}^{+}\left(t-t^{\prime}\right)\right] .
\end{array}
$$

Finally let us consider a powder or a single crystal with isotropic hyperfine structure. We must replace the product of rotation matrices by its average value :

$$
\sum_{p q}\left\langle D_{M q}^{L^{*}}\left(\theta_{\mathrm{i}} \varphi_{\mathrm{i}}\right) \mathfrak{D}_{M^{\prime} p}^{L}\left(\theta_{\mathrm{i}} \varphi_{\mathrm{i}}\right)\right\rangle_{\theta_{\mathrm{i}} \varphi_{\mathrm{i}}}=\frac{8 \pi}{2 L+1} \delta_{M, M^{\prime}}
$$

and we arrive at the final formula :

$$
\begin{aligned}
\left\langle V_{\text {diff }}\right\rangle=-\frac{i}{\hbar} \frac{8 \pi}{2 L+1} \int_{-\infty}^{t} \exp \left\{\left[-i\left(\Omega-\omega_{\mathrm{i}_{0}}\right)-\frac{\Gamma+\Gamma^{\prime}}{2}\right]\left(t-t^{\prime}\right)\right\} \mathrm{d} t^{\prime} \times \\
\quad \times \underset{\text { at }}{\operatorname{Trace}}\left\{\mathbf{T}_{L}^{+} U_{0}\left(t-t^{\prime}\right) \mathbf{T}_{L} \sigma U_{0}^{+}\left(t-t^{\prime}\right)\right\} .
\end{aligned}
$$

Since we have assumed that $|f\rangle$ and $|g\rangle$ were eigenfunctions of $\mathscr{H}_{0}$ and $U_{0}$, this can also be written in explicit form :

$$
\left\langle V_{\text {diff }}\right\rangle=\frac{i}{\hbar} \frac{8 \pi}{2 L+1} \sum_{f_{1} g_{1}} \sigma_{g_{1} g_{1}} \frac{\left\langle g_{1}\left|\mathbf{T}_{L}^{+}\right| f_{1}\right\rangle\left\langle f_{1}\left|\mathbf{T}_{L}\right| g_{1}\right\rangle}{-i\left(\Omega-\omega_{\mathrm{i}_{0}}\right)+i\left(\omega_{g_{1}}-\omega_{f_{1}}\right)-\frac{\Gamma+\Gamma^{\prime}}{2}} .
$$

Notice that the real part of the refraction index as deduced from eq. (25) and (35) is an increasing function of the frequency as one expects.

Notice also that éq. (34) is in agreement with eq. (II.8), (II .10) and (II.11) of Cohen-Tannoudji and Laloe [11]. In terms of the Liouville evolution superoperator [4] :

$$
\vartheta_{0}(t)=\exp \left(-\frac{i}{\hbar} \mathfrak{H}_{0}^{\times} t\right)
$$

corresponding to $U_{0}(t)$ the trace in eq. (34) can also be expressed as :

$$
\sum \sigma_{g_{1} g_{1}}\left\langle f_{1}\left|\mathbf{T}_{L}\right| g_{1}\right\rangle\left\langle g_{2}\left|\mathbf{T}_{L}^{+}\right| f_{2}\right\rangle\left\langle f_{2} g_{2}\left|\vartheta_{0}\left(t-t^{\prime}\right)\right| f_{1} g_{1}\right\rangle .
$$

We will use this result when we introduce the relaxation in part II. 
4. Application to Mössbauer scattering. - 4.1 General FORMULAS. - We are interested in the number of photons $\mathrm{d} n_{\mathbf{k} \lambda} / \mathrm{d} t$ scattered per second as a function of the number $n_{\mathbf{k}_{\mathbf{i}} \lambda_{\mathbf{i}}}$ of incident photons in the mode $\mathbf{k}_{\mathbf{i}} \lambda_{\mathbf{i}}$. Starting from eq. (19) and making $\tilde{\mathscr{H}}_{\mathbf{R}}$ explicit we arrive at :

$$
\begin{aligned}
& \frac{\mathrm{d} n_{\mathbf{k} \lambda}}{\mathrm{d} t}=\left(\frac{2 \pi \hbar c}{L^{3}}\right)^{2} \frac{1}{k_{\mathrm{i}} k} n_{\mathbf{k}_{\mathbf{i}} \lambda_{\mathrm{i}}} \times \frac{1}{\hbar^{4}} \sum_{\substack{f^{\prime} g^{\prime \prime}, g^{\prime \prime} \\
\int_{-\infty}}}^{t} \mathrm{~d} t^{\prime} \int_{-\infty}^{t} \mathrm{~d} t^{\prime \prime} \int_{-\infty}^{t^{\prime \prime}} \mathrm{d} t^{\prime \prime \prime} \exp \left[i\left(\omega_{\mathbf{k} \lambda}-\Omega\right)\left(t-t^{\prime \prime}\right)\right] \times \\
& \times \exp \left[-i\left(\omega_{\mathbf{k}_{\mathbf{i}} \lambda_{\mathrm{i}}}-\Omega\right)\left(t^{\prime}-t^{\prime \prime \prime}\right)\right] \exp \left[-\frac{\Gamma}{2}\left(t-t^{\prime}\right)\right] \exp \left[-\frac{\Gamma}{2}\left(t^{\prime \prime}-t^{\prime \prime \prime}\right)\right] \\
& \times\left\langle g\left|U_{0}^{+}(t) H_{\mathrm{a}}^{+}(\mathbf{k}, \lambda) U_{0}(t)\right| f^{\prime}\right\rangle\left\langle f^{\prime}\right| U_{0}^{+}\left(t^{\prime}\right) H_{\mathrm{a}}\left(\mathbf{k}_{\mathrm{i}} \lambda_{\mathrm{i}}\right) \\
& \times\left(U_{0}\left(t^{\prime}\right)\left|g^{\prime}\right\rangle b_{g^{\prime}}\left(t^{\prime}\right) b_{g^{\prime \prime}}^{*}\left(t^{\prime \prime \prime}\right)\left\langle g^{\prime \prime}\right| U_{0}^{+}\left(t^{\prime \prime \prime}\right)\right) \\
& \times H_{\mathrm{a}}^{+}\left(k_{\mathrm{i}} \lambda_{\mathrm{i}}\right) U_{0}\left(t^{\prime \prime \prime}\right)\left|f^{\prime \prime}\right\rangle\left\langle f^{\prime \prime}\left|U_{0}^{+}\left(t^{\prime \prime}\right) H_{\mathrm{a}}(\mathbf{k}, \lambda) U_{0}\left(t^{\prime \prime}\right)\right| g\right\rangle+\text { c.c. . (36) }
\end{aligned}
$$

In the interaction representation we have that :

$$
\sum_{g^{\prime} g^{\prime \prime}}\left|g^{\prime}\right\rangle b_{g^{\prime}}\left(t^{\prime}\right) b_{g^{\prime \prime}}^{*}\left(t^{\prime \prime \prime}\right)\left\langle g^{\prime \prime}|=| \psi\left(t^{\prime}\right)\right\rangle\left\langle\psi\left(t^{\prime \prime \prime}\right)\right|
$$

On the other hand the wave function in the normal representation is :

$$
\psi(t)=U_{0}(t) \psi\left(t^{\prime}\right) .
$$

Consequently the parenthesis in the middle of eq. (36) can be put into the form :

$$
\sum_{g^{\prime} g^{\prime \prime}} U_{0}\left(t^{\prime}\right)\left|g^{\prime}\right\rangle b_{g^{\prime}}\left(t^{\prime}\right) b_{g^{\prime \prime}}^{*}\left(t^{\prime \prime \prime}\right) U_{0}^{+}\left(t^{\prime \prime \prime}\right) \equiv\left|\psi\left(t^{\prime}\right)\right\rangle\left\langle\psi\left(t^{\prime \prime \prime}\right)\right|=U_{0}\left(t^{\prime}-t^{\prime \prime \prime}\right) \sigma\left(t^{\prime \prime \prime}\right)=\sigma\left(t^{\prime}\right) U_{0}^{+}\left(t^{\prime \prime \prime}-t^{\prime}\right)
$$

where $\sigma$ is the density matrix of the ground state of the atom in the normal representation. In practice it is the Boltzmann matrix :

$$
\sigma\left(t^{\prime}\right)=\sigma\left(t^{\prime \prime \prime}\right)=\sigma_{\mathbf{B}} .
$$

Eq. (36) is relative to a monochromatic irradiation. If we irradiate with a lorentzian distribution we have to consider the integral :

$$
\frac{1}{\pi} \int_{-\infty}^{+\infty} \mathrm{d} \omega_{\mathbf{k}_{\mathbf{i}} \lambda_{\mathrm{i}}} \frac{\Gamma^{\prime} / 2}{\left(\omega_{\mathbf{k}_{\mathbf{i}} \lambda_{\mathrm{i}}}-\omega_{\mathrm{i}_{0}}\right)^{2}+\left(\Gamma^{\prime} / 2\right)^{2}} \exp \left[-i\left(\omega_{\mathbf{k}_{\mathbf{i}} \lambda_{\mathrm{i}}}-\Omega\right)\left(t^{\prime}-t^{\prime \prime \prime}\right)\right]
$$

The way of performing the contour integration depends on the sign of $\left(t^{\prime}-t^{\prime \prime \prime}\right)$.

If $t^{\prime}-t^{\prime \prime \prime}>0$ the integral is equal to :

$$
\exp \left\{\left[-i\left(\omega_{\mathrm{i}_{0}}-\Omega\right)-\frac{\Gamma^{\prime}}{2}\right]\left(t^{\prime}-t^{\prime \prime \prime}\right)\right\}
$$

If $t^{\prime}-t^{\prime \prime \prime}<0$ it is equal to :

$$
\exp \left\{\left[+i\left(\omega_{\mathrm{i}_{0}}-\Omega\right)-\frac{\Gamma^{\prime}}{2}\right]\left(t^{\prime \prime \prime}-t^{\prime}\right)\right\}
$$

In the triple time integral of eq. (36) we must therefore order the times.

This can be achieved by writing :

$$
\begin{gathered}
\int_{-\infty}^{t} \mathrm{~d} t^{\prime} \int_{-\infty}^{t} \mathrm{~d} t^{\prime \prime} \int_{-\infty}^{t^{\prime \prime}} \mathrm{d} t^{\prime \prime \prime}=\int_{-\infty}^{t} \mathrm{~d} t^{\prime} \int_{-\infty}^{t^{\prime}} \mathrm{d} t^{\prime \prime} \int_{-\infty}^{t^{\prime \prime}} \mathrm{d} t^{\prime \prime \prime}+\int_{-\infty}^{t} \mathrm{~d} t^{\prime \prime} \int_{-\infty}^{t^{\prime \prime}} \mathrm{d} t^{\prime} \int_{-\infty}^{t^{\prime}} \mathrm{d} t^{\prime \prime \prime}+ \\
I=I_{1}+I_{2}+I_{3} .
\end{gathered}
$$

In view of the subsequent introduction of relaxation we will also rearrange the trace in each integral according to the prescriptions of appendix I of [4]. This consists in putting it into the form

$$
\operatorname{Trace}\left\{U_{0}\left(t-t_{n}\right) A U_{0}\left(t_{n}-t_{n-1}\right) B \ldots U_{0}\left(t_{2}-t_{1}\right) \sigma C U_{0}^{+}\left(t_{2}-t_{1}\right) D \ldots U_{0}^{+}\left(t-t_{n}\right) F\right\}
$$


with time ordered $U_{0}$ on the left of $\sigma$ and corresponding $U_{0}^{+}$on the right. By making use of eq. (39) and dropping for simplicity the polarization index, we arrive at :

$$
\begin{aligned}
& I_{1}=\int_{-\infty}^{t} \mathrm{~d} t^{\prime} \int_{-\infty}^{t^{\prime}} \mathrm{d} t^{\prime \prime} \int_{-\infty}^{t^{\prime \prime}} \mathrm{d} t^{\prime \prime \prime} \exp \left\{\left[i\left(\omega_{k}-\Omega\right)-\frac{\Gamma}{2}\right]\left(t-t^{\prime}\right)\right\} \exp \left\{\left[i\left(\omega_{k}-\omega_{\mathrm{i}_{0}}\right)-\frac{\Gamma^{\prime}}{2}\right]\left(t^{\prime}-t^{\prime \prime}\right)\right\} \times \\
& \times \exp \left\{\left[-i\left(\omega_{\mathrm{i}_{0}}-\Omega\right)-\frac{\Gamma+\Gamma^{\prime}}{2}\right]\left(t^{\prime \prime}-t^{\prime \prime \prime}\right)\right\} \underset{\text { at }}{\operatorname{Trace}}\left\{H_{\mathrm{a}}^{+}(\mathbf{k}) U_{0}\left(t-t^{\prime}\right) H_{\mathbf{a}}\left(\mathbf{k}_{\mathrm{i}}\right) U_{0}\left(t^{\prime}-t^{\prime \prime}\right)\right. \\
& \left.\times U_{0}\left(t^{\prime \prime}-t^{\prime \prime \prime}\right) \sigma\left(t^{\prime \prime \prime}\right) H_{\mathrm{a}}^{+}\left(\mathbf{k}_{\mathrm{i}}\right) U_{0}^{+}\left(t^{\prime \prime}-t^{\prime \prime \prime}\right) H_{\mathrm{a}}(\mathbf{k}) U_{0}^{+}\left(t^{\prime}-t^{\prime \prime}\right) U_{0}^{+}\left(t-t^{\prime}\right)\right\} \\
& I_{2}=\int_{-\infty}^{t} \mathrm{~d} t^{\prime \prime} \int_{-\infty}^{t^{\prime \prime}} \mathrm{d} t^{\prime} \int_{-\infty}^{t^{\prime}} \mathrm{d} t^{\prime \prime \prime} \exp \left\{\left[i\left(\omega_{k}-\Omega\right)-\frac{\Gamma}{2}\right]\left(t-t^{\prime}\right)\right\} \mathrm{e}^{-\Gamma\left(t^{\prime \prime}-t^{\prime}\right)} \times \\
& \times \exp \left\{\left[-i\left(\omega_{\mathrm{i}_{0}}-\Omega\right)-\frac{\Gamma+\Gamma^{\prime}}{2}\right]\left(t^{\prime}-t^{\prime \prime \prime}\right)\right\} \underset{\mathrm{at}}{\operatorname{Trace}}\left\{H_{\mathrm{a}}^{+}(\mathbf{k}) U_{0}\left(t-t^{\prime \prime}\right) U_{0}\left(t^{\prime \prime}-t^{\prime}\right)\right. \\
& \left.\times\left[H_{\mathrm{a}}\left(\mathbf{k}_{\mathrm{i}}\right) U_{0}\left(t^{\prime}-t^{\prime \prime \prime}\right) \sigma\left(t^{\prime \prime \prime}\right) H_{\mathrm{a}}^{+}\left(\mathbf{k}_{\mathrm{i}}\right) U_{0}^{+}\left(t^{\prime}-t^{\prime \prime \prime}\right)\right] U_{0}^{+}\left(t^{\prime \prime}-t^{\prime}\right) H_{\mathbf{a}}(\mathbf{k}) U_{0}^{+}\left(t-t^{\prime \prime}\right)\right\} \\
& I_{3}=\int_{-\infty}^{t} \mathrm{~d} t^{\prime \prime} \int_{-\infty}^{t^{\prime \prime}} \mathrm{d} t^{\prime \prime \prime} \int_{-\infty}^{t^{\prime \prime \prime}} \mathrm{d} t^{\prime} \exp \left\{\left[i\left(\omega_{k}-\Omega\right)-\frac{\Gamma}{2}\right]\left(t-t^{\prime \prime}\right)\right\} \mathrm{e}^{-\Gamma\left(t^{\prime \prime}-t^{\prime \prime \prime}\right)} \times \\
& \times \exp \left\{\left[i\left(\omega_{\mathrm{i}_{0}}-\Omega\right)-\frac{\Gamma+\Gamma^{\prime}}{2}\right]\left(t^{\prime \prime \prime}-t^{\prime}\right)\right\} \underset{\mathrm{at}}{\operatorname{Trace}}\left\{H_{\mathrm{a}}^{+}(\mathbf{k}) U_{0}\left(t-t^{\prime \prime}\right) U_{0}\left(t^{\prime \prime}-t^{\prime \prime \prime}\right)\right. \\
& \left.\times\left[U_{0}\left(t^{\prime \prime \prime}-t^{\prime}\right) H_{\mathrm{a}}\left(\mathbf{k}_{\mathrm{i}}\right) \sigma\left(t^{\prime}\right) U_{0}^{+}\left(t^{\prime \prime \prime}-t^{\prime}\right) H_{\mathrm{a}}^{+}\left(\mathbf{k}_{\mathrm{i}}\right)\right] U_{0}^{+}\left(t^{\prime \prime}-t^{\prime \prime \prime}\right) H_{\mathrm{a}}(\mathbf{k}) U_{0}^{+}\left(t-t^{\prime \prime}\right)\right\} .
\end{aligned}
$$

It is possible [4] to express these traces in terms of Liouville evolution superoperators

$$
\vartheta_{0}=\exp \left(-\frac{i}{\hbar} \mathscr{H}_{0}^{\times} t\right)
$$

Trace $\mathrm{n}^{0} 1=\sum\left[\left\langle g_{4}\left|H_{\mathrm{a}}^{+}(k)\right| f_{4}\right\rangle\left\langle f_{1}\left|H_{\mathrm{a}}\left(\mathbf{k}_{\mathrm{j}}\right)\right| g_{3}\right\rangle \sigma_{g_{1} g_{1}}\left\langle g_{6}\left|H_{\mathrm{a}}^{+}(\mathbf{k})\right| f_{5}\right\rangle \times\left\langle f_{7}\left|H_{\mathrm{a}}^{+}(\mathbf{k})\right| g_{7}\right\rangle\right] \times$

$$
\times \delta_{g_{1} g_{6}}\left\langle f_{4} g_{4}\left|v_{0}\left(t-t^{\prime}\right)\right| f_{1} g_{8}\right\rangle\left\langle g_{3} g_{8}\left|v_{0}\left(t^{\prime}-t^{\prime \prime}\right)\right| g_{2} g_{7}\right\rangle\left\langle g_{2} f_{7}\left|v_{0}\left(t^{\prime \prime}-t^{\prime \prime \prime}\right)\right| g_{1} f_{5}\right\rangle \text {. }
$$

Trace no $2=\sum$ [same bracket] $\times \delta_{g_{1} g_{6}}\left\langle f_{4} g_{4}\left|\vartheta_{0}\left(t-t^{\prime \prime}\right)\right| f_{3} g_{7}\right\rangle\left\langle f_{3} f_{7}\left|\vartheta_{0}\left(t^{\prime \prime}-t^{\prime}\right)\right| f_{1} f_{6}\right\rangle$

$$
\left\langle g_{3} f_{6}\left|\vartheta_{0}\left(t^{\prime}-t^{\prime \prime \prime}\right)\right| g_{1} f_{5}\right\rangle \text {. }
$$

Trace no $3=\sum$ [same bracket] $\times \delta_{g_{3} g_{1}} \times\left\langle f_{4} g_{4}\left|v_{0}\left(t-t^{\prime \prime}\right)\right| f_{3} g_{7}\right\rangle\left\langle f_{3} f_{7}\left|v_{0}\left(t^{\prime \prime}-t^{\prime \prime \prime}\right)\right| f_{2} f_{5}\right\rangle$

$$
\left\langle f_{2} g_{6}\left|\mho_{0}\left(t^{\prime \prime \prime}-t^{\prime}\right)\right| f_{1} g_{1}\right\rangle \text {. }
$$

We shall use these complicated expressions when we introduce relaxation. In the absence of relaxation, since we assumed $|f\rangle$ and $|g\rangle$ to be eigenstates of $\mathcal{H}_{0}$, things simplify and we arrive at :

$$
\frac{\mathrm{d} n_{k}}{\mathrm{~d} t} \propto 2 \operatorname{Re} I=2 \operatorname{Re}\left(I_{1}+I_{2}+I_{3}\right)
$$

$$
\begin{aligned}
I_{1}+I_{2} & +I_{3}=-\sum_{g_{1} g_{4}}\left\langle g_{4}\left|H_{\mathrm{a}}^{+}(\mathbf{k})\right| f_{1}\right\rangle\left\langle f_{1}\left|H_{\mathrm{a}}\left(\mathbf{k}_{\mathrm{i}}\right)\right| g_{1}\right\rangle \sigma_{g_{1} g_{1}} \times\left\langle g_{1}\left|H_{\mathrm{a}}^{+}\left(\mathbf{k}_{\mathrm{i}}\right)\right| f_{5}\right\rangle\left\langle f_{5}\left|H_{\mathrm{a}}(\mathbf{k})\right| g_{4}\right\rangle \times \\
& \times\left\{\left[\frac{1}{i\left(\omega_{k}-\Omega\right)-\frac{\Gamma}{2}-i\left(\omega_{f_{1}}-\omega_{g_{4}}\right)} \times \frac{1}{i\left(\omega_{k}-\omega_{\mathrm{i}_{0}}\right)-i\left(\omega_{g_{1}}-\omega_{g_{4}}\right)-\frac{\Gamma^{\prime}}{2}} \times \frac{1}{-i\left(\omega_{\mathrm{i}_{0}}-\Omega\right)-\frac{\Gamma+\Gamma^{\prime}}{2}-i\left(\omega_{g_{1}}-\omega_{f_{5}}\right)}\right]\right. \\
& +\left[\frac{1}{i\left(\omega_{k}-\Omega\right)-\frac{\Gamma}{2}-i\left(\omega_{f_{1}}-\omega_{g_{4}}\right)} \times \frac{1}{-\Gamma-i\left(\omega_{f_{1}}-\omega_{f_{5}}\right)} \times \frac{1}{-i\left(\omega_{\mathrm{i}_{0}}-\Omega\right)-\frac{\Gamma+\Gamma^{\prime}}{2}-i\left(\omega_{g_{1}}-\omega_{f_{5}}\right)}\right] \\
& \left.+\left[\frac{1}{i\left(\omega_{k}-\Omega\right)-\frac{\Gamma}{2}-i\left(\omega_{f_{1}}-\omega_{g_{4}}\right)} \times \frac{1}{-\Gamma-i\left(\omega_{f_{1}}-\omega_{f_{5}}\right)} \times \frac{1}{+i\left(\omega_{\mathrm{i}_{0}}-\Omega\right)-\frac{\Gamma+\Gamma^{\prime}}{2}-i\left(\omega_{f_{1}}-\omega_{g_{1}}\right)}\right]\right\}
\end{aligned}
$$

As a check for this expression we may investigate what it becomes in three limiting situations $a$ ) $\Gamma^{\prime} \rightarrow \infty$ (irradiation with a white spectrum), $b$ ) $\Gamma^{\prime} \rightarrow 0$ (monochromatic irradiation and $c$ ) irradiation far from resonance. 
4.2. IrRAdiation With a White SPeCtrum $\left(\Gamma^{\prime} \rightarrow \infty\right)$. - In this case the bracket in eq. (52) reduces to :

$$
\frac{2}{\Gamma^{\prime}} \frac{1}{i\left(\omega_{k}-\Omega\right)-\frac{\Gamma}{2}-i\left(\omega_{f_{1}}-\omega_{g_{4}}\right)} \times \frac{1}{-\Gamma-i\left(\omega_{f_{1}}-\omega_{f_{5}}\right)}
$$

and $I$ itself has a very simple interpretation which is most apparent when one returns to eq. (36). If we integrate over $\omega_{k_{1} \lambda_{1}}$ :

$$
\int \exp \left[-i\left(\omega_{k_{1} \lambda_{1}}-\Omega\right)\left(t^{\prime}-t^{\prime \prime \prime}\right)\right] \mathrm{d} \omega_{k_{1} \lambda_{i}}=2 \pi \delta\left(t^{\prime}-t^{\prime \prime \prime}\right) .
$$

Then eq. (36) takes the form

$$
\begin{aligned}
\frac{\mathrm{d} n_{k \lambda}}{\mathrm{d} t} \propto & \operatorname{Re} 2 \pi \int_{-\infty}^{t} \mathrm{~d} t^{\prime \prime} \int_{-\infty}^{t^{\prime \prime}} \mathrm{d} t^{\prime \prime \prime} \exp \left\{\left[i\left(\omega_{k \lambda}-\Omega\right)-\frac{\Gamma}{2}\right]\left(t-t^{\prime \prime}\right)\right\} \mathrm{e}^{-\Gamma\left(t^{\prime \prime}-t^{\prime \prime \prime}\right)} \times \\
& \times \underset{\text { a }}{\operatorname{Trace}}\left\{H_{\mathrm{a}}^{+}(\mathbf{k}) U_{0}\left(t-t^{\prime \prime}\right) U_{0}\left(t^{\prime \prime}-t^{\prime \prime \prime}\right) H_{\mathrm{a}}\left(\mathbf{k}_{\mathrm{i}}\right) \sigma\left(t^{\prime \prime \prime}\right) H_{\mathrm{a}}^{+}\left(\mathbf{k}_{\mathrm{i}}\right) U_{0}^{+}\left(t^{\prime \prime}-t^{\prime \prime \prime}\right) H_{\mathrm{a}}(\mathbf{k}) U_{0}^{+}\left(t-t^{\prime \prime}\right)\right\} .
\end{aligned}
$$

In this expression :

$\sigma\left(t^{\prime \prime \prime}\right)=\sigma_{\mathrm{B}}$ is the density matrix of the ground state, $\sigma_{\mathrm{in}}^{\mathrm{I}} \equiv H_{\mathrm{a}}\left(\mathbf{k}_{\mathrm{i}}\right) \sigma\left(t^{\prime \prime \prime}\right) H_{\mathrm{a}}^{+}\left(\mathbf{k}_{\mathrm{i}}\right)$ is the initial density matrix of the excited state when it is populated by the incident radiation, $\sigma^{\prime}\left(t^{\prime \prime}-t^{\prime \prime \prime}\right) \equiv U_{0}\left(t^{\prime \prime}-t^{\prime \prime \prime}\right) \sigma_{\text {in }}^{\mathrm{I}} U_{0}^{+}\left(t^{\prime \prime}-t^{\prime \prime \prime}\right)$ is what this matrix has become after a time interval $t^{\prime \prime}-t^{\prime \prime \prime}$,

$$
\sigma_{\mathrm{ev}}^{\mathrm{I}}(1 / \Gamma)=\int_{-\infty}^{t^{\prime \prime}} \mathrm{d} t^{\prime \prime \prime} \mathrm{e}^{-\Gamma\left(t^{\prime \prime}-t^{\prime \prime \prime}\right)} \sigma^{\mathrm{I}}\left(t^{\prime \prime}-t^{\prime \prime \prime}\right)
$$

is the average density matrix of state $\mathrm{I}$ at the time of emission of the scattered $\gamma$ ray. In terms of $\sigma_{\mathrm{ev}}^{\mathrm{I}}(1 / \Gamma)$ :

$$
\begin{aligned}
\frac{\mathrm{d} n_{k}}{\mathrm{~d} t} \propto \operatorname{Re} 2 \pi \int_{-\infty}^{t} \mathrm{~d} t^{\prime \prime} \exp \left\{\left[i\left(\omega_{k}-\Omega\right)-\frac{\Gamma}{2}\right]\left(t-t^{\prime \prime}\right)\right\} \times \\
\times \operatorname{Trace}\left\{H_{\mathrm{a}}^{+}(\mathbf{k}) U_{0}\left(t-t^{\prime \prime}\right) \sigma_{\mathrm{ev}}^{\mathrm{I}}(1 / \Gamma) H_{\mathrm{a}}(\mathbf{k}) U_{0}^{+}\left(t-t^{\prime \prime}\right)\right\} .
\end{aligned}
$$

More precisely if we write $H_{\mathrm{a}}(\mathbf{k})$ in explicit form, it is easy to check that for a powder, we have a scattered intensity :

$$
I(\omega) \propto \frac{\mathrm{d} n_{k}}{\mathrm{~d} t} \propto \operatorname{Re} \int_{0}^{\infty} \mathrm{d} \tau \operatorname{Trace}\left\{\mathbf{T}_{L}(0) \mathbf{T}_{L}^{+}(\tau) \sigma_{\mathrm{ev}}^{\mathrm{I}}(1 / \Gamma)\right\} \exp \left\{\left[i\left(\omega_{k}-\Omega\right)-\frac{\Gamma}{2}\right] \tau\right\}
$$

This equation is identical to eq. (1) relative to an emitter (in (1), $\omega \equiv \omega_{k}-\Omega$ ) except for the fact that here we may vary $\sigma_{\mathrm{ev}}^{\mathrm{I}}(1 / \Gamma)$ by changing the parameters of the incident radiation (for example its polarization), while in a emitter $\sigma^{\mathrm{I}}(1 / \Gamma)$ is uniquely determined as long as we do not detect the first step of the cascade (as in PAC).

These conclusions are in agreement with eq. (53) in which the first fraction describes the emission of the scattered photon, while the second one describes the evolution of $\sigma_{f_{1} f_{s}}$ in the excited state. In conventional Mössbauer spectroscopy irradiation with a white spectrum is not feasable, but it could perhaps become possible by use of synchrotron radiation [12].

4.3 Monochromatic IRRAdiation $\left(\Gamma^{\prime} \rightarrow 0\right)$. - This case too is not physical but it will enable us to check that in this limit, we do recover the results of a standard second order probability calculation for resonant scattering, in particular as it concerns the energy conservation between the initial state and the final state [19]. Such a calculation would lead to :

$$
\frac{\mathrm{d} n_{k}}{\mathrm{~d} t} \propto \frac{2 \pi}{\hbar^{2}} n_{\mathrm{i}} \sum_{g_{1} g_{4}} \sigma_{g_{1} g_{1}}\left|\sum_{f_{1}} \frac{\left\langle g_{4}\left|H_{\mathrm{a}}^{+}(\mathbf{k})\right| f_{1}\right\rangle\left\langle f_{1}\left|H_{\mathrm{a}}\left(\mathbf{k}_{\mathrm{i}}\right)\right| g_{1}\right\rangle}{\omega_{\mathrm{i}_{0}}-\Omega+\omega_{g_{1}}-\omega_{f_{1}}+i \frac{\Gamma}{2}}\right|^{2} \times \delta\left(\omega_{k}+\omega_{g_{4}}-\omega_{\mathrm{i}_{0}}-\omega_{g_{1}}\right) .
$$


For checking this point, let us return to the bracket of eq. (52) and add the last two lines; we get :

$$
\begin{aligned}
\frac{1}{i\left(\omega_{k}-\Omega\right)-\frac{\Gamma}{2}-i\left(\omega_{f_{1}}-\omega_{g_{4}}\right)} \times \frac{1}{-i\left(\omega_{\mathrm{i}_{0}}-\Omega\right)-\frac{\Gamma+\Gamma^{\prime}}{2}-i\left(\omega_{g_{1}}-\omega_{f_{5}}\right)} \\
\times \frac{1}{i\left(\omega_{\mathrm{i}_{0}}-\Omega\right)-i\left(\omega_{f_{1}}-\omega_{g_{1}}\right)-\frac{\Gamma+\Gamma^{\prime}}{2}} \times\left[1+\frac{\Gamma^{\prime}}{\Gamma+i\left(\omega_{f_{1}}-\omega_{f_{5}}\right)}\right] .
\end{aligned}
$$

When we insert this expression into the bracket of eq. (52) it finally becomes :

$$
\begin{aligned}
\{\} & =\left[\frac{1}{i\left(\omega_{\mathrm{i}_{0}}-\Omega\right)-i\left(\omega_{f_{1}}-\omega_{g_{1}}\right)-\frac{\Gamma+\Gamma^{\prime}}{2}} \frac{1}{i\left(\omega_{k}-\omega_{\mathrm{i}_{0}}\right)-i\left(\omega_{g_{1}}-\omega_{g_{5}}\right)-\frac{\Gamma^{\prime}}{2}} \times \frac{1}{-i\left(\omega_{\mathrm{i}_{0}}-\Omega\right)-\frac{\Gamma+\Gamma^{\prime}}{2}-i\left(\omega_{g_{1}}-\omega_{f_{5}}\right)}\right]+ \\
& +\Gamma^{\prime}\left[\frac{1}{i\left(\omega_{k}-\Omega\right)-\frac{\Gamma}{2}-i\left(\omega_{f_{1}}-\omega_{g_{4}}\right)} \times \frac{1}{-i\left(\omega_{\mathrm{i}_{0}}-\Omega\right)-\frac{\Gamma+\Gamma^{\prime}}{2}-i\left(\omega_{g_{1}}-\omega_{f_{5}}\right)} \times \frac{1}{i\left(\omega_{\mathrm{i}_{0}}-\Omega\right)-i\left(\omega_{f_{1}}-\omega_{g_{1}}\right)-\frac{\Gamma+\Gamma^{\prime}}{2}}\right. \\
& \left.\times\left(\frac{1}{\Gamma+i\left(\omega_{f_{1}}-\omega_{f_{5}}\right)}-\frac{1}{i\left(\omega_{k}-\omega_{\mathrm{i}_{0}}\right)-i\left(\omega_{g_{1}}-\omega_{g_{4}}\right)-\frac{\Gamma^{\prime}}{2}}\right)\right] .
\end{aligned}
$$

When $\Gamma^{\prime} \rightarrow 0$, in the first part [ ] of this expression, the first and third fractions remain finite and the second one reduces to :

$$
-i \mathcal{T} \frac{1}{\omega_{k}-\omega_{\mathrm{i}_{0}}-\left(\omega_{g_{1}}-\omega_{g_{4}}\right)}-\pi \delta\left(\omega_{k}-\omega_{\mathrm{i}_{0}}-\left(\omega_{g_{1}}-\omega_{g_{4}}\right)\right)
$$

In the second part [ ], which is multiplied by $\Gamma^{\prime}$, all fractions remain finite, except for the last one whose limit is minus eq. (61). If we take account of the multiplicative factor $\Gamma^{\prime}$, it follows that when $\Gamma^{\prime} \rightarrow 0$, eq. (60) reduces to its first part [ ]. Inserting this contribution in eq. (52) we easily recover eq. (58).

4.4 IRRADIATION FAR FROM RESONANCE. - Let us now assume that $\Gamma^{\prime} \sim \Gamma$, that the absorber has a well resolved absorption spectrum and that we irradiate far from any resonance

$$
\left.\begin{array}{l}
\omega_{\mathrm{i}_{0}}-\Omega-\left(\omega_{f_{1}}-\omega_{g_{1}}\right) \\
\omega_{\mathrm{i}_{0}}-\Omega-\left(\omega_{f_{5}}-\omega_{g_{1}}\right) \\
\omega_{\mathrm{i}_{0}}-\Omega-\left(\omega_{f_{1}}-\omega_{g_{4}}\right)
\end{array}\right\} \gg \Gamma, \Gamma^{\prime} .
$$

The bracket $\{\quad\}$ in eq. (52) then becomes :

$$
\begin{aligned}
& \{\}=\frac{1}{\left(\omega_{\mathrm{i}_{0}}-\Omega-\omega_{f_{1}}+\omega_{g_{1}}\right)\left(\omega_{\mathrm{i}_{0}}-\Omega-\omega_{f_{5}}+\omega_{g_{1}}\right)} \times \\
& \times\left[\frac{1}{i\left(\omega_{k}-\omega_{\mathrm{i}_{0}}-\omega_{g_{1}}+\omega_{g_{4}}\right)-\frac{\Gamma^{\prime}}{2}}+\left(\frac{\Gamma^{\prime}}{i\left(\omega_{k}-\Omega-\omega_{f_{1}}+\omega_{g_{4}}\right)-\frac{\Gamma}{2}} \times \frac{1}{\Gamma+i\left(\omega_{f_{1}}-\omega_{f_{5}}{ }^{\prime}\right.}\right)\right. \\
& \left.+\left(\frac{\Gamma^{\prime}}{i\left(\omega_{k}-\Omega-\omega_{f_{1}}+\omega_{g_{4}}\right)-\frac{\Gamma}{2}} \times \frac{1}{i\left(\omega_{k}-\omega_{i_{0}}-\omega_{g_{1}}+\omega_{g_{4}}\right)-\frac{\Gamma^{\prime}}{2}}\right)\right] .
\end{aligned}
$$

In the square bracket the maximum absolute value of the first term is $1 /\left(\Gamma^{\prime} / 2\right)$. For the second term, if $\omega_{f_{1}}=\omega_{f_{s}}$ its maximum value is $\Gamma^{\prime} /\left(\Gamma \times \Gamma^{\prime} / 2\right) \sim 1 / \Gamma^{\prime} / 2$; otherwise by virtue of our assumption concerning the well resolved character of the spectrum, it is much smaller. Finally the maximum value of the last term is :

$$
\frac{\Gamma^{\prime}}{\omega_{\mathrm{i}_{0}}-\Omega-\omega_{f_{1}}+\omega_{g_{1}}} \times \frac{1}{\Gamma / 2}
$$


i.e. also much smaller than the first term. Notice that for non-degenerate levels $\omega_{f_{1}}=\omega_{f_{5}}$ implies that $f_{1}=f_{5}$. In this case when we irradiate far from resonance :

$$
\begin{aligned}
\frac{\mathrm{d} n_{k}}{\mathrm{~d} t} \propto \sum_{g_{1} g_{4}}\left|\sum_{f_{1}} \frac{\left\langle g_{4}\left|H_{\mathrm{a}}^{+}(\mathbf{k})\right| f_{1}\right\rangle\left\langle f_{1}\left|H_{\mathrm{a}}\left(\mathbf{k}_{\mathrm{i}}\right)\right| g_{1}\right\rangle}{\omega_{\mathrm{i}_{0}}-\Omega-\omega_{f_{1}}+\omega_{g_{1}}}\right|^{2} \frac{\Gamma^{\prime} / 2}{\left(\omega_{k}-\omega_{\mathrm{i}_{0}}-\omega_{g_{1}}+\omega_{g_{4}}\right)^{2}+\frac{\Gamma^{\prime 2}}{4}}+ \\
+\frac{\Gamma^{\prime}}{\Gamma} \sum_{g_{1} g_{4}} \sigma_{g_{1} g_{1}} \sum_{f_{1}}\left|\frac{\left\langle g_{4}\left|H_{\mathrm{a}}^{+}(\mathbf{k})\right| f_{1}\right\rangle\left\langle f_{1}\left|H_{\mathrm{a}}\left(\mathbf{k}_{\mathrm{i}}\right)\right| g_{1}\right\rangle}{\omega_{\mathrm{i}_{0}}-\Omega-\omega_{f_{1}}+\omega_{g_{1}}}\right|^{2} \frac{\Gamma / 2}{\left(\omega_{k}-\Omega-\omega_{f_{1}}+\omega_{g_{4}}\right)^{2}+\frac{\Gamma^{2}}{4}} .
\end{aligned}
$$

In this equation the first contribution corresponds to Rayleigh $\left(g_{1}=g_{4}\right)$ and Raman $\left(g_{1} \neq g_{4}\right)$ scattering and the second one (which vanishes when $\Gamma^{\prime}=0$ ) to resonant emission from the weakly excited levels $f$. When there are only one level $f$ and one level $g$ and when $\Gamma^{\prime}=\Gamma$, the scattered spectrum is composed of one Rayleigh line and one resonant line with equal widths and intensities, in agreement with the classical calculation of Moon mentioned by Boyle and Hall ([13] p. 458).

4.5 Remarks. - When the ground and excited Mossbauer states are singlets the real part of the bracket in eq. (52) takes the form :

$$
-\frac{\Gamma^{\prime}}{2} \cdot \frac{1}{\left(\omega_{k}-\Omega\right)^{2}+\frac{\Gamma^{2}}{4}} \cdot \frac{1}{\left(\omega_{k}-\omega_{\mathrm{i}_{0}}\right)^{2}+\frac{\Gamma^{\prime 2}}{4}}
$$

in agreement with the eq. (3.5) of Boyle and Hall [13].

- In the absence of relaxation the incident linewidth could have been introduced in a much simpler way, by starting from eq. (58) and integrating over a Lorentzian profile with width $\Gamma^{\prime} / 2$. However, in the presence of relaxation we find it safer to use the time ordered integrals eq. (45-47) and to apply the prescriptions of appendix I of [4].

- With regard to Afanasev and Gorobchenko [7], they consider only the case of monochromatic irradiation i.e. they start from a formula of type (58) which they rearrange into an expression similar to our eq. (52) (with $\Gamma^{\prime}=0$ ).

5. Angular selection rules for scattering by a powder. - In this paragraph we will slightly correct some results of Balko and Hoy and compute some numerical values which we will use in the examples of part II.

Let us go back to eq. (52) and write the various $H_{\mathrm{a}}(\mathbf{k})$ in explicit form. There appears an angular factor :

$$
\begin{aligned}
\left\langle g_{4}\left|H_{\mathrm{a}}^{+}(\mathbf{k})\right| f_{1}\right\rangle\left\langle f_{1}\right| & H_{\mathrm{a}}\left(\mathbf{k}_{\mathrm{i}}\right)\left|g_{1}\right\rangle \sigma_{g_{1} g_{1}}\left\langle g_{1}\left|H_{\mathrm{a}}^{+}\left(\mathbf{k}_{\mathrm{i}}\right)\right| f_{5}\right\rangle\left\langle f_{5}\left|H_{\mathrm{a}}(\mathbf{k})\right| g_{4}\right\rangle \\
\propto & \sum_{p q} \mathcal{D}_{M^{\prime} q}^{L^{*}}(\mathbf{k}) \mathcal{D}_{M q}^{L}(\mathbf{k}) \mathcal{D}_{M^{\prime \prime \prime} p}^{L^{*}}\left(\mathbf{k}_{\mathrm{i}}\right) \mathscr{D}_{M^{\prime \prime} p}^{L}\left(\mathbf{k}_{\mathrm{i}}\right) \\
& = \pm 1 \\
\times & \left\langle g_{4}\left|T_{L M^{\prime}}^{+}\right| f_{1}\right\rangle\left\langle f_{1}\left|T_{L M^{\prime \prime}}\right| g_{1}\right\rangle \sigma_{g_{1} g_{1}}\left\langle g_{1}\left|T_{L M^{\prime \prime \prime}}^{+}\right| f_{5}\right\rangle\left\langle f_{5}\left|T_{L M}\right| g_{4}\right\rangle .
\end{aligned}
$$

We will denote the polar angles of $\mathbf{k}_{\mathbf{i}}$ and $\mathbf{k}$ respectively by $\left(\theta_{1} \varphi_{1}\right)$ and $\left(\theta_{2} \varphi_{2}\right)$ and define :

$$
P_{M^{\prime} M}(\theta, \varphi)=\sum_{q= \pm 1} \mathfrak{D}_{M^{\prime} q}^{L^{*}}(\theta, \varphi) \mathfrak{D}_{M q}^{L}(\theta, \varphi) .
$$

When the powder is composed of cristallites and when the atomic hamiltonian referred to the local axes of the crystallite is the same for all crystallites, we must average eq. (65) over $\left(\theta_{1} \varphi_{1}\right)$ and $\left(\theta_{2} \varphi_{2}\right)$ while keeping the angle of $\mathbf{k}_{\mathbf{i}}$ and $\mathbf{k}$ constant. In what follows we will only consider a scattering angle $\left(\mathbf{k}_{\mathbf{i}}, \mathbf{k}\right)=\pi / 2$. We must therefore compute (Messiah's conventions) :

$$
\begin{aligned}
& \left\langle P_{M^{\prime} M^{\prime}}^{L}\left(\theta_{2} \varphi_{2}\right) P_{M^{\prime \prime \prime} M^{\prime \prime}}^{L}\left(\theta_{1} \varphi_{1}\right)\right\rangle\left(\left(\mathbf{k}_{\mathrm{i}}, \mathbf{k}\right)=\pi / 2\right) \\
& =\sum_{p q}\left\langle\exp \left[-i\left(M-M^{\prime}\right) \varphi_{2}\right] \exp \left[-i\left(M^{\prime \prime}-M^{\prime \prime \prime}\right) \varphi_{1}\right] r_{M^{\prime} q}^{L}\left(\theta_{2}\right) r_{M q}^{L}\left(\theta_{2}\right) \times\right. \\
& \left.\times r_{M^{\prime \prime \prime} p}^{L}\left(\theta_{1}\right) r_{M^{\prime \prime} p}^{L}\left(\theta_{1}\right)\right\rangle\left(\mathbf{k}_{\mathrm{i}}, \mathbf{k}=\frac{\pi}{2}\right) .
\end{aligned}
$$


For this we define a system of axe $O x y z$ attached to a crystallite, and another system $O X Y Z$ with Euler angles $\alpha$, $\beta, \gamma$ with respect to the first one. We take $\mathbf{k}_{\mathrm{i}}$ along $O Z$ and $\mathbf{k}$ along $O X$, so that $\left(\theta_{1} \varphi_{1}\right),\left(\theta_{2} \varphi_{2}\right)$ are functions of $(\alpha, \beta, \gamma)$ and we average over $\alpha(0 \rightarrow 2 \pi), \beta(0 \rightarrow \pi)$ and $\gamma(0 \rightarrow 2 \pi)$.

It is very easy to show that eq. (67) is non-zero only if

$$
M-M^{\prime}+M^{\prime \prime}-M^{\prime \prime \prime}=0 \text {. }
$$

As for the numerical values of the averages, they must be computed for each value of $L$. We will consider two examples :

$L=1$ : for example magnetic dipole radiation $\left({ }^{57} \mathrm{Fe}\right)$ with arbitrary local hamiltonian. The only non-zero averages in this case are :

$$
\begin{gathered}
\left\langle P_{11}(\mathbf{k}) P_{11}\left(\mathbf{k}_{\mathrm{i}}\right)\right\rangle=\left\langle P_{11}(\mathbf{k}) P_{-1-1}\left(\mathbf{k}_{\mathrm{i}}\right)\right\rangle=\left\langle P_{-1-1}(\mathbf{k}) P_{11}\left(\mathbf{k}_{\mathrm{i}}\right)\right\rangle=\left\langle P_{-1-1}(\mathbf{k}) P_{-1-1}\left(\mathbf{k}_{\mathrm{i}}\right)\right\rangle=\frac{13}{30} \\
\left\langle P_{00}(\mathbf{k}) P_{00}\left(\mathbf{k}_{\mathrm{i}}\right)\right\rangle=\frac{12}{30} \\
\left\langle P_{11}(\mathbf{k}) P_{00}\left(\mathbf{k}_{\mathrm{i}}\right)\right\rangle=\left\langle P_{-1-1}(\mathbf{k}) P_{00}\left(\mathbf{k}_{\mathrm{i}}\right)\right\rangle=\left\langle P_{00}(\mathbf{k}) P_{11}\left(\mathbf{k}_{\mathrm{i}}\right)\right\rangle=\left\langle P_{00}(\mathbf{k}) P_{-1-1}\left(\mathbf{k}_{\mathrm{i}}\right)\right\rangle=\frac{14}{30} \\
\left\langle P_{10} P_{01}\right\rangle=\left\langle P_{0-1} P_{-10}\right\rangle=-\left\langle P_{10} P_{-10}\right\rangle=-\left\langle P_{0-1} P_{01}\right\rangle=-\frac{1}{30} \\
\left\langle P_{1-1} P_{-11}\right\rangle=\left\langle P_{-11} P_{1-1}\right\rangle=-\frac{1}{15}
\end{gathered}
$$

The first three lines, which correspond to very simple selection rules, are in agreement with the results of Balko and Hoy [5]. The other terms were not taken into account by these authors. If, as a first approximation, we take the first three lines as all equal to $13 / 30$, it is legitimate to neglect the mixed terms of the last two lines. However if we distinguish between $12 / 30,13 / 30,14 / 30$, we must keep the mixed term.

$L=2$ with isotropic hyperfine structure, for example $\mathrm{Yb}^{170} \mathrm{Au}$ (electric quadrupole nuclear transition).

In this case we may take the direction of $\mathbf{k}_{\mathrm{i}}$ as the $z$ axis for all crystallites, $\mathbf{k}$ then rotates in the plane $O x y$ perpendicular to $O z$. The coefficients $r_{M q}^{L}$ for $L=2$ can be found in reference [14]. It is found that the only non-zero averages are :

$$
\left\langle P_{11}\left(\mathbf{k}_{\mathrm{i}}\right) P_{11}(\mathbf{k})\right\rangle=\left\langle P_{11}\left(\mathbf{k}_{\mathrm{i}}\right) P_{-1-1}(\mathbf{k})\right\rangle=\left\langle P_{11}\left(\mathbf{k}_{\mathrm{i}}\right) P_{22}(\mathbf{k})\right\rangle=\left\langle P_{11}\left(\mathbf{k}_{\mathrm{i}}\right) P_{-2-2}(\mathbf{k})\right\rangle=\frac{1}{2}
$$

(and similar expressions with $P_{-1-1}\left(\mathbf{k}_{\mathrm{i}}\right)$ ). We will use these results in the next chapter.

6. Final remark. - Let us mention that prior to this work several authors had studied the influence of the incident radiation linewidth on the apparent Landé factor of the excited Mössbauer state [15-17]. With the development of very narrow band lasers, similar investigations are now being undertaken [18] in the optical range; a difference with the Mössbauer case is that the laser linewidth is usually controlled by its instabilities and is therefore gaussian and not lorentzian. In the case $\Gamma^{\prime} \sim \Gamma$ this may seriously influence the physical phenomena. A good illustration of this point is given by the Hanle effect : consider an atom with an excited state $J=1$ and a ground state $J_{g}=0$ in a magnetic field $H$ parallel to $O z$. We irradiate it along $O y$ with light polarized parallel to $O x$ and we observe the intensities $I_{x}, I_{y}$ scattered along $O z$ with linear polarizations $O x, O y$. Let $P=\frac{I_{x}-I_{4}}{I_{x}+I_{y}}$. In a Hanlé experiment one measures the variation $\Delta P$ of $P$ when $H$ varies from zero to infity. Let us assume that the incident radiation is centered on the transition $m_{J}=0 \rightarrow m_{J}=0$ and has a width $\Gamma^{\prime}$.

If the incident profile is lorentzian, $\Delta P$ as a function of $g_{J} \mu_{\mathrm{B}} H$ is lorentzian with a constant width $\Gamma$ but its total amplitude, $\Delta P^{T}=\Delta P(H=0)-\Delta P(H=\infty)$, depends on $\Gamma^{\prime}$ :

$$
\frac{\Delta P_{\Gamma^{\prime}=\infty}^{T}}{1}=\frac{\Delta P_{\Gamma^{\prime}=\Gamma}^{T}}{3 / 2}=\frac{\Delta P_{\Gamma^{\prime}=0}^{T}}{2}=1 .
$$

On the contrary if the profile is gaussian and if $\Gamma^{\prime} \lesssim$ a few $\Gamma, \Delta P$ has a constant amplitude $\Delta P^{T}=2$, but a varying width. These behaviours are quite different. 


\section{References}

[1] GonZalez-Jimenez, F., Imbert, P., Hartmann-Boutron, F., Phys. Rev. B 9 (1974) 95.

[2] Hartmann-Boutron, F., Phys. Rev. B 10 (1974) 2113.

[3] Gonzalez-Jimenez, F., Hartmann-Boutron, F., Imbert, P., Phys. Rev. B 10 (1974) 2122.

[4] Hartmann-Boutron, F., Spanjaard, D., J. Physique 36 (1975) 605.

[5] Balko, B., Hoy, G. R., Phys. Rev. B 10 (1974) 36

[6] Balko, B., Hoy, G. R., Phys. Rev. B 10 (1974) 4523.

[7] Afanasev, A. M., Gorobchenko, V. D., Zh. T.E.F. 67 (1974) 2246.

[8] Hartmann-Boutron, F., Spanjaard, D., J. Phys. 33 (1972) 285.

[9] Barrat, J. P., Cohen-Tannoudj, C., J. Physique 22 (1961) 329; J. Physique 22 (1961) 443

[10] Blume, M., Kistner, O. C., Phys. Rev. 171 (1968) 417.
[11] Cohen-Tannoudji, C., Laloe, F., J. Physique 28 (1967) 505.

[12] Ruby, S. L., J. Physique Colloq. 36 (1975) C6-209.

[13] Boyle, A. J. F., Hall, H. E., The Mössbauer effect in Rep. Prog. Phys. 25 (1962) 441.

[14] Haroche, S., Ann. Physique 6 (1971) 327 (p. 373).

[15] Goebel, C. G., McVoy, K. W., Phys. Rev. 148 (1966) 1021.

[16] Eicher, H., Z. Phys. 212 (1968) 176.

[17] Ehehalt, R., Nolte, E., Körner, H. J., Wagner, F. E., Kiente, P., Communication to the International Conference on the Applications of the Mössbauer Effect, Ayelet Hashahar, Israel, August 1972.

[18] Rasmussen, W., Schieder, R., Walther, M., Opt. Commun. 12 (1974) 315 and references therein.

[19] HeItler, H., The Quantum Theory of Radiation (Oxford Clarendon Press. Third Edition) 1960. 